\title{
A classifier-based approach to preposition and determiner error correction in L2 English
}

\author{
Rachele De Felice and Stephen G. Pulman \\ Oxford University Computing Laboratory \\ Wolfson Building, Parks Road, Oxford OX1 3QD, UK \\ \{rachele.defelice|stephen.pulman\}@comlab.ox.ac.uk
}

\begin{abstract}
In this paper, we present an approach to the automatic identification and correction of preposition and determiner errors in nonnative (L2) English writing. We show that models of use for these parts of speech can be learned with an accuracy of $70.06 \%$ and $92.15 \%$ respectively on L1 text, and present first results in an error detection task for L2 writing.
\end{abstract}

\section{Introduction}

The field of research in natural language processing (NLP) applications for L2 language is constantly growing. This is largely driven by the expanding population of L2 English speakers, whose varying levels of ability may require different types of NLP tools from those designed primarily for native speakers of the language. These include applications for use by the individual and within instructional contexts. Among the key tools are error-checking applications, focusing particularly on areas which learners find the most challenging. Prepositions and determiners are known to be one of the most frequent sources of error for L2 English speakers, a finding supported by our analysis of a small error-tagged corpus we created (determiners $17 \%$ of errors, prepositions $12 \%$ ). Therefore, in developing a system for automatic error detection in L2 writing, it seems desirable to focus on these problematic, and very common, parts of speech (POS).

This paper gives a brief overview of the problems posed by these POS and of related work. We

(c) 2008 . Licensed under the Creative Commons Attribution-Noncommercial-Share Alike 3.0 Unported license (http://creativecommons.org/licenses/by-nc-sa/3.0/). Some rights reserved. then present our proposed approach on both L1 and L2 data and discuss the results obtained so far.

\section{The problem}

\subsection{Prepositions}

Prepositions are challenging for learners because they can appear to have an idiosyncratic behaviour which does not follow any predictable pattern even across nearly identical contexts. For example, we say I study in Boston but I study at MIT; or He is independent of his parents, but dependent on his son. As it is hard even for L1 speakers to articulate the reasons for these differences, it is not surprising that learners find it difficult to master prepositions.

\subsection{Determiners}

Determiners pose a somewhat different problem from prepositions as, unlike them, their choice is more dependent on the wider discourse context than on individual lexical items. The relation between a noun and a determiner is less strict than that between a verb or noun and a preposition, the main factor in determiner choice being the specific properties of the noun's context. For example, we can say boys like sport or the boys like sport, depending on whether we are making a general statement about all boys or referring to a specific group. Equally, both she ate an apple and she ate the apple are grammatically well-formed sentences, but only one may be appropriate in a given context, depending on whether the apple has been mentioned previously. Therefore, here, too, it is very hard to come up with clear-cut rules predicting every possible kind of occurrence. 


\section{Related work}

Although in the past there has been some research on determiner choice in L1 for applications such as generation and machine translation output, work to date on automatic error detection in L2 writing has been fairly limited. Izumi et al. (2004) train a maximum entropy classifier to recognise various errors using contextual features. They report results for different error types (e.g. omission - precision $75.7 \%$, recall $45.67 \%$; replacement - P $31.17 \%$, $\mathrm{R} 8 \%$ ), but there is no break-down of results by individual POS. Han et al. (2006) use a maximum entropy classifier to detect determiner errors, achieving 83\% accuracy. Chodorow et al. (2007) present an approach to preposition error detection which also uses a model based on a maximum entropy classifier trained on a set of contextual features, together with a rule-based filter. They report $80 \%$ precision and $30 \%$ recall. Finally, Gamon et al. (2008) use a complex system including a decision tree and a language model for both preposition and determiner errors, while Yi et al. (2008) propose a web count-based system to correct determiner errors (P 62\%, R 41\%).

The work presented here displays some similarities to the papers mentioned above in its use of a maximum entropy classifier and a set of features. However, our feature set is more linguistically sophisticated in that it relies on a full syntactic analysis of the data. It includes some semantic components which we believe play a role in correct class assignment.

\section{Contextual models for prepositions and determiners}

\subsection{Feature set}

The approach proposed in this paper is based on the belief that although it is difficult to formulate hard and fast rules for correct preposition and determiner usage, there is enough underlying regularity of characteristic syntactic and semantic contexts to be able to predict usage to an acceptable degree of accuracy. We use a corpus of grammatically correct English to train a maximum entropy classifier on examples of correct usage. The classifier can therefore learn to associate a given preposition or determiner to particular contexts, and reliably predict a class when presented with a novel instance of a context for one or the other.

The L1 source we use is the British National

\begin{tabular}{|l|l|} 
Head noun & 'apple' \\
Number & singular \\
Noun type & count \\
Named entity? & no \\
WordNet category & food, plant \\
Prep modification? & yes, 'on' \\
Object of Prep? & no \\
Adj modification? & yes, 'juicy' \\
Adj grade & superlative \\
POS \pm 3 & VV, DT, JJS, IN, DT, NN \\
\hline
\end{tabular}

Table 1: Determiner feature set for Pick the juiciest apple on the tree.

\begin{tabular}{|l|l|}
\hline POS modified & verb \\
Lexical item modified & 'drive' \\
WordNet Category & motion \\
Subcat frame & pp_to \\
POS of object & noun \\
Object lexical item & 'London' \\
Named entity? & yes, type = location \\
POS \pm 3 & NNP, VBD, NNP \\
Grammatical relation & iobj \\
\hline
\end{tabular}

Table 2: Preposition feature set for John drove to London.

Corpus (BNC) as we believe this offers a representative sample of different text types. We represent training and testing items as vectors of values for linguistically motivated contextual features. Our feature vectors include 18 feature categories for determiners and 13 for prepositions; the main ones are illustrated in Table 1 and Table 2 respectively. Further determiner features note whether the noun is modified by a predeterminer, possessive, numeral, and/or a relative clause, and whether it is part of a 'there is...' phrase. Additional preposition features refer to the grade of any adjectives or adverbs modified (base, comparative, superlative) and to whether the items modified are modified by more than one $\mathrm{PP}^{1}$.

In De Felice and Pulman (2007), we described some of the preprocessing required and offered some motivation for this approach. As for our choice of features, we aim to capture all the elements of a sentence which we believe to have an effect on preposition and determiner choice, and which can be easily extracted automatically - this is a key consideration as all the features derived rely on automatic processing of the text. Grammatical relations refer to RASP-style grammatical relations between heads and complements in which the preposition occurs (see e.g. (Briscoe et al.,

\footnotetext{
${ }^{1}$ A full discussion of each feature, including motivation for its inclusion and an assessment of its contribution to the model, is found in De Felice (forthcoming).
} 


\begin{tabular}{|l|l|}
\hline Author & Accuracy \\
\hline Baseline & $26.94 \%$ \\
Gamon et al. 08 & $64.93 \%$ \\
Chodorow et al. 07 & $69.00 \%$ \\
Our model & $\mathbf{7 0 . 0 6 \%}$ \\
\hline
\end{tabular}

Table 3: Classifier performance on L1 prepositions

2006)). Semantic word type information is taken from WordNet lexicographer classes, 40 broad semantic categories which all nouns and verbs in WordNet belong to ${ }^{2}$ (e.g. 'verb of motion', 'noun denoting food'), while the POStags are from the Penn Treebank tagset - we note the POS of three words either side of the target word ${ }^{3}$. For each occurrence of a preposition or determiner in the corpus, we obtain a feature vector consisting of the preposition or determiner and its context, described in terms of the features noted above.

\section{Acquiring the models}

\subsection{Prepositions}

At the moment, we restrict our analysis to the nine most frequent prepositions in the data: $\boldsymbol{a t}, \boldsymbol{b y}, \boldsymbol{f o r}$, from, in, of, on, to, and with, to ensure a sufficient amount of data for training. This gives a training dataset comprising 8,898,359 instances. We use a standard maximum entropy classifier ${ }^{4}$ and do not omit any features, although we plan to experiment with different feature combinations to determine if, and how, this would impact the classifier's performance. Before testing our model on learner data, it is important to ascertain that it can correctly associate prepositions to a given context in grammatical, well-edited data. We therefore tested the model on a section of the BNC not used in training, section J. Our best result to date is $\mathbf{7 0 . 0 6 \%}$ accuracy (test set size: 536,193). Table 3 relates our results to others reported in the literature on comparable tasks. The baseline refers to always choosing the most frequent option, namely $\boldsymbol{o} f$.

We can see that our model's performance compares favourably to the best results in the literature, although direct comparisons are hard to draw since different groups train and test on different preposition sets and on different types of data (British vs. American English, BNC vs. news reports, and so

\footnotetext{
${ }^{2}$ No word sense disambiguation was performed at this stage.

${ }^{3}$ In NPs with a null determiner, the target is the head noun.

${ }^{4}$ Developed by James Curran.
}

\begin{tabular}{|l|r|l|l|}
\hline & Proportion of training data & Precision & Recall \\
\hline of & $27.83 \%(2,501,327)$ & $74.28 \%$ & $90.47 \%$ \\
to & $20.64 \%(1,855,304)$ & $85.99 \%$ & $81.73 \%$ \\
in & $17.68 \%(1,589,718)$ & $60.15 \%$ & $67.60 \%$ \\
for & $8.01 \%(720,369)$ & $55.47 \%$ & $43.78 \%$ \\
on & $6.54 \%(587,871)$ & $58.52 \%$ & $45.81 \%$ \\
with & $6.03 \%(541,696)$ & $58.13 \%$ & $46.33 \%$ \\
at & $4.72 \%(424,539)$ & $57.44 \%$ & $52.12 \%$ \\
by & $4.69 \%(421,430)$ & $63.83 \%$ & $56.51 \%$ \\
from & $3.86 \%(347,105)$ & $59.20 \%$ & $32.07 \%$ \\
\hline
\end{tabular}

Table 4: L1 results - individual prepositions

on). Furthermore, it should be noted that Gamon et al. report more than one figure in their results, as there are two components to their model: one determining whether a preposition is needed, and the other deciding what the preposition should be. The figure reported here refers to the latter task, as it is the most similar to the one we are evaluating. Additionally, Chodorow et al. also discuss some modifications to their model which can increase accuracy; the result noted here is the one more directly comparable to our own approach.

\subsubsection{Further discussion}

To fully assess the model's performance on the L1 data, it is important to consider factors such as performance on individual prepositions, the relationship between training dataset size and accuracy, and the kinds of errors made by the model.

Table 4 shows the classifier's performance on individual prepositions together with the size of their training datasets. At first glance, a clear correlation appears between the amount of data seen in training and precision and recall, as evidenced for example by $\boldsymbol{o f}$ or to, for which the classifier achieves a very high score. In other cases, however, the correlation is not so clear-cut. For example by has one of the smallest data sets in training but higher scores than many of the other prepositions, while for is notable for the opposite reason, namely having a large dataset but some of the lowest scores.

The absence of a definite relation between dataset size and performance suggests that there might be a cline of 'learnability' for these prepositions: different prepositions' contexts may be more or less uniquely identifiable, or they may have more or fewer senses, leading to less confusion for the classifier. One simple way of verifying the latter case is by looking at the number of senses assigned to the prepositions by a resource 


\begin{tabular}{|r|l|r|r|r|r|r|r|r|r|}
\hline Target prep & \multicolumn{6}{l|}{ Confused with } \\
\hline & at & by & for & from & in & of & on & to & with \\
\hline at & $\mathrm{xx}$ & $4.65 \%$ & $10.82 \%$ & $2.95 \%$ & $36.83 \%$ & $19.46 \%$ & $9.17 \%$ & $10.28 \%$ & $5.85 \%$ \\
\hline by & $6.54 \%$ & $\mathrm{xx}$ & $8.50 \%$ & $2.58 \%$ & $41.38 \%$ & $19.44 \%$ & $5.41 \%$ & $10.04 \%$ & $6.10 \%$ \\
\hline for & $8.19 \%$ & $3.93 \%$ & $\mathrm{xx}$ & $1.91 \%$ & $25.67 \%$ & $36.12 \%$ & $5.60 \%$ & $11.29 \%$ & $7.28 \%$ \\
\hline from & $6.19 \%$ & $4.14 \%$ & $6.72 \%$ & $\mathrm{xx}$ & $26.98 \%$ & $26.74 \%$ & $7.70 \%$ & $16.45 \%$ & $5.07 \%$ \\
\hline in & $7.16 \%$ & $9.28 \%$ & $10.68 \%$ & $3.01 \%$ & $\mathrm{xx}$ & $43.40 \%$ & $10.92 \%$ & $8.96 \%$ & $6.59 \%$ \\
\hline of & $3.95 \%$ & $2.00 \%$ & $18.81 \%$ & $3.36 \%$ & $40.21 \%$ & $\mathrm{xx}$ & $9.46 \%$ & $14.77 \%$ & $7.43 \%$ \\
\hline on & $5.49 \%$ & $3.85 \%$ & $8.66 \%$ & $2.29 \%$ & $32.88 \%$ & $27.92 \%$ & $\mathrm{xx}$ & $12.20 \%$ & $6.71 \%$ \\
\hline to & $9.77 \%$ & $3.82 \%$ & $11.49 \%$ & $3.71 \%$ & $24.86 \%$ & $27.95 \%$ & $9.43 \%$ & $\mathrm{xx}$ & $8.95 \%$ \\
\hline with & $3.66 \%$ & $4.43 \%$ & $12.06 \%$ & $2.24 \%$ & $28.08 \%$ & $26.63 \%$ & $6.81 \%$ & $16.10 \%$ & $\mathrm{xx}$ \\
\hline
\end{tabular}

Table 5: Confusion matrix for L1 data - prepositions

such as the Oxford English Dictionary. However, we find no good correlation between the two as the preposition with the most senses is of (16), and that with the fewest is from (1), thus negating the idea that fewer senses make a preposition easier to learn. The reason may therefore be found elsewhere, e.g. in the lexical properties of the contexts.

A good picture of the model's errors can be had by looking at the confusion matrix in Table 5 , which reports, for each preposition, what the classifier's incorrect decision was. Analysis of these errors may establish whether they are related to the dataset size issue noted above, or have a more linguistically grounded explanation.

From the table, the frequency effect appears evident: in almost every case, the three most frequent wrong choices are the three most frequent prepositions, $\boldsymbol{t o}, \boldsymbol{o f}$, and $\boldsymbol{i n}$, although interestingly not in that order, in usually being the first choice. Conversely, the less frequent prepositions are less often suggested as the classifier's choice. This effect precludes the possibility at the moment of drawing any linguistic conclusions. These may only be gleaned by looking at the errors for the three more frequent prepositions. We see for example that there seems to be a strong relation between of and for, the cause of which is not immediately clear: perhaps they both often occur within noun phrases (e.g. book of recipes, book for recipes). More predictable is the confusion between to and from, and between locative prepositions such as to and $\boldsymbol{a t}$, although the effect is less strong for other potentially confusable pairs such as in and at on .

Table 6 gives some examples of instances where the classifier's chosen preposition differs from that found in the original text. In most cases, the classifier's suggestion is also grammatically correct,

\begin{tabular}{|l|l|}
\hline Classifier choice & Correct phrase \\
\hline demands of the sector & demands for... \\
condition for development & condition of... \\
travel to speed & travel at... \\
look at the USA & look to... \\
\hline
\end{tabular}

Table 6: Examples of classifier errors on preposition $\mathrm{L} 1$ task

\begin{tabular}{|l|l|}
\hline Author & Accuracy \\
\hline Baseline & $59.83 \%$ \\
Han et al. 06 & $83.00 \%$ \\
Gamon et al. 08 & $86.07 \%$ \\
Turner and Charniak 07 & $86.74 \%$ \\
Our model & $\mathbf{9 2 . 1 5 \%}$ \\
\hline
\end{tabular}

Table 7: Classifier performance - L1 determiners

but the overall meaning of the phrases changes somewhat. For example, while the demands of the sector are usually made by the sector itself, the demands for the sector suggest that someone else may be making them. These are subtle differences which it may be impossible to capture without a more sophisticated understanding of the wider context.

The example with travel, on the other hand, yields an ungrammatical result. We assume that the classifier has acquired a very strong link between the lexical item travel and the preposition to that directs it towards this choice (cf. also the example of look at/to). This suggests that individual lexical items play an important role in preposition choice along with other more general syntactic and semantic properties of the context. 


\begin{tabular}{|l|r|l|l|}
\hline & \%of training data & Prec. & Recall \\
\hline a & $9.61 \%(388,476)$ & $70.52 \%$ & $53.50 \%$ \\
the & $29.19 \%(1,180,435)$ & $85.17 \%$ & $91.51 \%$ \\
null & $61.20 \%(2,475,014)$ & $98.63 \%$ & $98.79 \%$ \\
\hline
\end{tabular}

Table 8: L1 results - individual determiners

\subsection{Determiners}

For the determiner task, we also consider only the three most frequent cases (a, the, null), which gives us a training dataset consisting of 4,043,925 instances. We achieve accuracy of $92.15 \%$ on the L1 data (test set size: 305,264 ), as shown in Table 7 . Again, the baseline refers to the most frequent class, null.

The best reported results to date on determiner selection are those in Turner and Charniak (2007). Our model outperforms their n-gram language model approach by over 5\%. Since the two approaches are not tested on the same data this comparison is not conclusive, but we are optimistic that there is a real difference in accuracy since the type of texts used are not dissimilar. As in the case of the prepositions, it is interesting to see whether this high performance is equally distributed across the three classes; this information is reported in Table 8 . Here we can see that there is a very strong correlation between amount of data seen in training and precision and recall. The indefinite article's lower 'learnability', and its lower frequency appears not to be peculiar to our data, as it is also found by Gamon et al. among others.

The disparity in training is a reflection of the distribution of determiners in the English language. Perhaps if this imbalance were addressed, the model would more confidently learn contexts of use for $\boldsymbol{a}$, too, which would be desirable in view of using this information for error correction. On the other hand, this would create a distorted representation of the composition of English, which may not be what we want in a statistical model of language. We plan to experiment with smaller scale, more similar datasets to ascertain whether the issue is one of training size or of inherent difficulty in learning about the indefinite article's occurrence.

In looking at the confusion matrix for determiners (Table 9), it is interesting to note that for the classifier's mistakes involving $\boldsymbol{a}$ or the the erroneous choice is in the almost always the other determiner rather than the null case. This suggests that the frequency effect is not so strong as to over-

\begin{tabular}{|r|l|l|c|}
\hline Target det & \multicolumn{4}{|l|}{ Confused with } \\
\hline & a & the & null \\
\hline $\mathbf{a}$ & $\mathrm{xx}$ & $92.92 \%$ & $7.08 \%$ \\
\hline the & $80.66 \%$ & $\mathrm{xx}$ & $19.34 \%$ \\
\hline null & $14.51 \%$ & $85.49 \%$ & $\mathrm{xx}$ \\
\hline
\end{tabular}

Table 9: Confusion matrix for $\mathrm{L} 1$ determiners

ride any true linguistic information the model has acquired, otherwise the predominant choice would always be the null case. On the contrary, these results show that the model is indeed capable of distinguishing between contexts which require a determiner and those which do not, but requires further fine tuning to perform better in knowing which of the two determiner options to choose. Perhaps the introduction of a discourse dimension might assist in this respect. We plan to experiment with some simple heuristics: for example, given a sequence 'Determiner Noun', has the noun appeared in the preceding few sentences? If so, we might expect the to be the correct choice rather than $\boldsymbol{a}$.

\section{Testing the model}

\subsection{Working with $\mathrm{L} 2$ text}

To evaluate the model's performance on learner data, we use a subsection of the Cambridge Learner Corpus (CLC) $)^{5}$. We envisage our model to eventually be of assistance to learners in analysing their writing and identifying instances of preposition or determiner usage which do not correspond to what it has been trained to expect; the more probable instance would be suggested as a more appropriate alternative. In using NLP tools and techniques which have been developed with and for L1 language, a loss of performance on L2 data is to be expected. These methods usually expect grammatically well-formed input; learner text is often ungrammatical, misspelled, and different in content and structure from typical L1 resources such as the WSJ and the BNC.

\subsection{Prepositions}

For the preposition task, we extract 2523 instances of preposition use from the CLC (1282 correct, 1241 incorrect) and ask the classifier to mark them

\footnotetext{
${ }^{5}$ The CLC is a computerised database of contemporary written learner English (currently over $25 \mathrm{~m}$ words). It was developed jointly by Cambridge ESOL and Cambridge University Press. The Cambridge Error Coding System has been developed and applied manually to the data by Cambridge University Press.
} 


\begin{tabular}{|l|l|}
\hline Instance type & Accuracy \\
\hline Correct & $66.7 \%$ \\
Incorrect & $70 \%$ \\
\hline
\end{tabular}

Table 10: Accuracy on L2 data - prepositions. Accuracy on incorrect instances refers to the classifier successfully identifying the preposition in the text as not appropriate for that context.

as correct or incorrect. The results from this task are presented in Table 10. These first results suggest that the model is fairly robust: the accuracy rate on the correct data, for example, is not much lower than that on the L1 data. In an application designed to assist learners, it is important to aim to reduce the rate of false alarms - cases where the original is correct, but the model flags an error - to a minimum, so it is positive that this result is comparatively high. Accuracy on error identification is at first glance even more encouraging. However, if we look at the suggestions the model makes to replace the erroneous preposition, we find that these are correct only $51.5 \%$ of the time, greatly reducing its usefulness.

\subsubsection{Further discussion}

A first analysis of the classifier's decisions and its errors points to various factors which could be impairing its performance. Spelling mistakes in the input are one of the most immediate ones. For example, in the sentence I'm Franch, responsable on the computer services, the classifier is not able to suggest a correct alternative to the erroneous on: since it does not recognise the adjective as a misspelling of responsible, it loses the information associated with this lexical feature, which could potentially determine the preposition choice.

A more complex problem arises when poor grammar in the input misleads the parser so that the information it gives for a sentence is incorrect, especially as regards PP attachment. In this example, I wold like following equipment to my speech: computer, modem socket and microphone, the missing the leads the parser to treat following as a verb, and believes it to be the verb to which the preposition is attached. It therefore suggests from as a correction, which is a reasonable choice given the frequency of phrases such as to follow from. However, this was not what the PP was meant to modify: impaired performance from the parser could be a significant negative factor in the model's performance. It would be interesting to test the model on texts written by students of different levels of proficiency, as their grammar may be more error-free and more likely to be parsed correctly. Alternatively, we could modify the parser so as to skip cases where it requires several attempts before producing a parse, as these more challenging cases could be indicative of very poorly structured sentences in which misused prepositions are dependent on more complex errors.

A different kind of problem impacting our accuracy scores derives from those instances where the classifier selects a preposition which can be correct in the given context, but is not the correct one in that particular case. In the example I received a beautiful present at my birthday, the classifier identifies the presence of the error, and suggests the grammatically and pragmatically appropriate correction for. The corpus annotators, however, indicate on as the correct choice. Since we use their annotations as the benchmark against which to evaluate the model, this instance is counted as the classifier being wrong because it disagrees with the annotators. A better indication of the model's performance may be to independently judge its decisions, to avoid being subject to the annotators' bias. Finally, we are beginning to look at the relations between preposition errors and other types of error such as verb choice, and how these are annotated in the data.

An overview of the classifier's error patterns for the data in this task shows that they are largely similar to those observed in the L1 data. This suggests that the gap in performance between L1 and L2 is due more to the challenges posed by learner text than by inherent shortcomings in the model, and therefore that the key to better performance is likely to lie in overcoming these problems. In future work we plan to use L2 data where some of the spelling errors and non-preposition or determiner errors have been corrected so that we can see which of the other errors are worth focussing on first.

\subsection{Determiners}

Our work on determiner error correction is still in the early stages. We follow a similar procedure to the prepositions task, selecting a number of both correct and incorrect instances. On the former (set size 2000) accuracy is comparable to that on L1 data: $\mathbf{9 2 . 2 \%}$. The danger of false alarms, then, appears not to be as significant as for the prepositions 
task. On the incorrect instances (set size ca. 1200), however, accuracy is less than $10 \%$.

Preliminary error analysis shows that the model is successful at identifying cases of misused determiner, e.g. $\boldsymbol{a}$ for the or vice versa, doing so in over two-thirds of cases. However, by far the most frequent error type for determiners is not confusion between indefinite and definite article, but omitting an article where one is needed. At the moment, the model detects very few of these errors, no doubt influenced by the preponderance of null cases seen in training. Furthermore, some of the issues raised earlier in discussing the application of NLP tools to L2 language hold for this task, too.

In addition to those, though, in this task more than for prepositions we believe that differences in text type between the training texts - the BNC and the testing material - learner essays - has a significant negative effect on the model. In this task, the lexical items play a crucial role in class assignment. If the noun in question has not been seen in training, the classifier may be unable to make an informed choice. Although the BNC comprises a wide variety of texts, there may not be a sufficient number covering topics typical of learner essays, such as 'business letters' or 'postcards to penpals'. Also, the BNC was created with material from almost 20 years ago, and learners writing in contemporary English may use lexical items which are not very frequently seen in the BNC. A clear example of this discrepancy is the noun internet, which requires the definite article in English, but not in several other languages, leading to countless sentences such as I saw it in internet, I booked it on internet, and so on. This is one of the errors the model never detects: a fact which is not surprising when we consider that this noun occurs only four times in the whole of the training data. It may be therefore necessary to consider using alternative sources of training data to overcome this problem and improve the classifier's performance.

\section{Comparison to human learners}

In developing this model, our first aim was not to create something which learns like a human, but something that works in the best and most efficient possible way. However, it is interesting to see whether human learners and classifiers display similar patterns of errors in preposition choice. This information has twofold value: as well as being of pedagogical assistance to instructors of En- glish L2, were the classifier to display student-like error patterns, insights into 'error triggers' could be derived from the L2 pedagogical literature to improve the classifier. The analysis of the types of errors made by human learners yields some insights which might be worthy of further investigation. A clear one is the confusion between the three locative and temporal prepositions at, in, and on (typical sentence: The training programme will start at the 1st August). This type of error is made often by both learners and the model on both types of data, suggesting that perhaps further attention to features might be necessary to improve discrimination between these three prepositions.

There are also interesting divergences. For example, a common source of confusion in learners is between by and from, as in I like it because it's from my favourite band. However, this confusion is not very frequent in the model, a difference which could be explained either by the fact that, as noted above, performance on from is very low and so the classifier is unlikely to suggest it, or that in training the contexts seen for $\boldsymbol{b y}$ are sufficiently distinctive that the classifier is not misled like the learners.

Finally, a surprising difference comes from looking at what to is confused with. The model often suggests at where to would be correct. This is perhaps not entirely unusual as both can occur with locative complements (one can go to a place or be at a place) and this similarity could be confusing the classifier. Learners, however, although they do make this kind of mistake, are much more hampered by the confusion between for and to, as in She was helpful for me or This is interesting for you. In other words, for learners it seems that the abstract use of this preposition, its benefactive sense, is much more problematic than the spatial sense. We can hypothesise that the classifier is less distracted by these cases because the effect of the lexical features is stronger.

A more detailed discussion of the issues arising from the comparison of confusion pairs cannot be had here. However, in noting both divergences and similarities between the two learners, human and machine, we may be able to derive useful insights into the way the learning processes operate, and what factors could be more or less important for them. 


\section{Conclusions and future directions}

This paper discussed a contextual feature based approach to the automatic acquisition of models of use for prepositions and determiners, which achieve an accuracy of $70.06 \%$ and $92.15 \%$ respectively, and showed how it can be applied to an error correction task for L2 writing, with promising early results. There are several directions that can be pursued to improve accuracy on both types of data. The classifier can be further fine-tuned to acquire more reliable models of use for the two POS. We can also experiment with its confidence thresholds, for example allowing it to make another suggestion when its confidence in its first choice is low. Furthermore, issues relating to the use of NLP tools with L2 data must be addressed, such as factoring out spelling or other errors in the data, and perhaps training on text types which are more similar to the CLC. In the longer term, we also envisage mining the information implicit in our training data to create a lexical resource describing the statistical tendencies observed.

\section{Acknowledgements}

We wish to thank Stephen Clark and Laura Rimell for stimulating discussions and the anonymous reviewers for their helpful comments. We acknowledge Cambridge University Press's assistance in accessing the Cambridge Learner Corpus data. Rachele De Felice was supported by an AHRC scholarship for the duration of her studies.

\section{References}

Briscoe, Ted, John Carroll, and Rebecca Watson. 2006. The second release of the RASP system. In COLING-ACL 06 Demo Session.

Chodorow, Martin, Joel Tetreault, and Na-Rae Han. 2007. Detection of grammatical errors involving prepositions. In Proceedings of the 4th $A C L$ SIGSEM Workshop on Prepositions.

De Felice, Rachele and Stephen Pulman. 2007. Automatically acquiring models of preposition use. In Proceedings of the 4th ACL-SIGSEM Workshop on Prepositions.

De Felice, Rachele. forthcoming. Recognising preposition and determiner errors in learner English. Ph.D. thesis, Oxford University Computing Laboratory.

Gamon, M., J. Gao, C. Brockett, A. Klementiev, W. Dolan, D. Belenko, and L. Vanderwende. 2008. Using contextual speller techniques and language modeling for ESL error correction. In Proceedings of IJCNLP.
Han, Na-Rae, Martin Chodorow, and Claudia Leacock. 2006. Detecting errors in English article usage by non-native speakers. Natural Language Engineering, 12(1):115-129.

Izumi, Emi, Kiyotaka Uchimoto, and Hitoshi Isahara. 2004. SST speech corpus of Japanese learners' English and automatic detection of learners' errors. ICAME, 28:31-48.

Turner, Jenine and Eugen Charniak. 2007. Language modeling for determiner selection. In NAACL-HLT Companion volume.

Yi, Xing, Jianfeng Gao, and William Dolan. 2008. A web-based English proofing system for ESL users. In Proceedings of IJCNLP. 